12,13

\title{
Плазмонная спектроскопия анизотропного отражения света от наночастиц металла, находящихся на поверхности полупроводника
}

\section{(C) В.А. Кособукин, А.В. Коротченков}

Физико-технический институт им. А.Ф. Иоффре РАН, Санкт-Петербург, Россия

E-mail: Vladimir.Kosobukin@mail.ioffe.ru

(Поступила в Редакцию 28 апреля 2016 г.)

\begin{abstract}
Представлена теория дифференциального анизотропного отражения света от наночастиц, обладающих плазмонами и находящихся вблизи границы раздела сред. Рассматривается модель монослоя одинаковых металлических частиц эллипсоидальной формы, заполняющих узлы прямоугольной решетки. Методом функций Грина в самосогласованном приближении квазиточечных диполей вычислены эффективные плазмонные поляризуемости наночастиц в слое. Учитывается эффект локального поля, обусловленный анизотропными дипольными плазмонами частиц слоя и диполями их изображения. Наблюдавшиеся недавно резонансные спектры анизотропного отражения света от нанокластеров индия на поверхности InAs объясняются различием частот, принадлежащих плазмонам с ортогональными поляризациями в плоскости поверхности. Показано, что различие плазмонных частот может быть связано с анизотропией формы частиц или/и структуры слоя, причем знак разности частот различен для этих двух типов анизотропии.
\end{abstract}

\section{1. Введение}

В последнее время значительное внимание уделяется поляризационным оптическим эффектам, в которых проявляется анизотропия плазмонов. Это относится к эллипсометрии $[1,2]$, магнитоплазмонике [3] и дифференциальной спектроскопии анизотропного отражения света [4-7]. В спектре анизотропного отражения света от нанокластеров индия, сформированных на поверхности (001) InAs [5,6], недавно наблюдалась резонансная спектральная особенность, обусловленная плазмонами. Эта особенность была связана с макроскопической анизотропией массива нанокластеров, которая не была выявлена обычными методами диагностики. Плазмонное происхождение обнаруженной спектральной особенности подтверждается ее красным смещением при увеличении размера наночастиц и диэлектрической проницаемости окружающей среды [6]. Масштаб плазмонного спектра анизотропного отражения, наблюдаемый для частиц индия на поверхности (001) GaAs [4] и InAs $[5,6]$, на один-два порядка величины превышает масштаб $\sim 10^{-3}$, характерный для спектров анизотропного отражения атомарных структур на поверхности полупроводников [8-10].

Теоретическая интерпретация наблюдаемых спектров анизотропного отражения проводилась в моделях эффективной среды [4] и регулярного массива наночастиц в слоистой диэлектрической среде $[3,6]$. Несмотря на концептуальное различие указанных подходов и различие полупроводниковых подложек (GaAs в [4] и InAs в $[5,6])$, из экспериментальных плазмонных спектров анизотропного отражения получены близкие оценки степени анизотропии массивов эллипсоидальных частиц индия. В работе [6] было показано также, что эффект струк- турной анизотропии массива, отсутствующий в модели эффективной среды, сравним с эффектом анизотропии формы частиц. Сходные аспекты плазмоники анизотропных систем затрагивались в ряде других работ [11-13]. Однако для интерпретации новых спектроскопических эффектов представляется актуальным развитие общей теории поляризационно-зависимых оптических эффектов с участием анизотропных плазмонов.

В настоящей работе представлена теория плазмонной спектроскопии анизотропного отражения света массивами металлических наночастиц. Принципиальной особенностью изучаемых систем является наличие анизотропного локального поля, обусловленного действием дипольных плазмонов наночастиц [3]. Обычно локальное поле значительно отличается от макроскопического, а его спектральные свойства и поляризация могут существенно зависеть от анизотропии резонансных диполей и их расположения [3,11-13]. Далее мы учитываем эффекты анизотропии формы наночастиц и структуры слоя, который они образуют. Резонансный оптический отклик с учетом локального поля плазмонов вычисляется самосогласованным образом в модели квазиточечных диполей, образующих прямоугольную решетку вблизи границы раздела сред.

Содержание статьи заключается в следующем. В разд. 2 обсуждается постановка задачи, в разд. 3 представлено ее общее решение с учетом эффекта локального поля, описанного в разд. 4. В разд. 5 вычислены наблюдаемые оптические величины, а их зависимость от анизотропии формы частиц и межчастичных расстояний обсуждается в разд. 6. Результаты численного анализа и оценка геометрических параметров анизотропии слоя наночастиц на основе наблюдаемых спектров приведены в разд. 7. 


\section{2. Постановка задачи и основные соотношения}

Задача решается методом функций Грина электродинамики $[3,14]$. Невозмущенной считается среда, образованная оптически однородными слоями макроскопической толщины, которые перпендикулярны оси $z$. Для этой среды компоненты тензора локальной изотропной диэлектрической проницаемости представляются в виде $\varepsilon_{\alpha \beta}^{(0)}(z, \omega)=\delta_{\alpha \beta} \varepsilon^{(0)}(z, \omega)$, где $\delta_{\alpha \beta}-$ символ Кронекера с декартовыми индексами $\alpha$ и $\beta, \omega-$ частота. Функция $\varepsilon^{(0)}(z, \omega)$, описывающая эффекты макроскопической электродинамики, равна $\varepsilon_{n}$ в $n$-м однородном слое.

Возмущением является поляризация $\mathbf{P}(z, \boldsymbol{\rho} ; \omega)$. Полное электрическое поле $\mathbf{E}(z, \boldsymbol{\rho} ; \omega)$ получается в результате решения последовательности уравнений

$$
\begin{aligned}
& \sum_{\nu} {\left[\sum_{\mu} \operatorname{rot}_{\alpha \mu} \operatorname{rot}_{\mu \nu}-\varepsilon^{(0)}(z ; \omega) k_{0}^{2} \delta_{\alpha \nu}\right] } \\
& \quad \times\left\{\begin{array}{c}
E_{v}^{(0)}(z, \boldsymbol{\rho}) \\
D_{v \beta}^{(0)}\left(z, z^{\prime}, \boldsymbol{\rho}-\boldsymbol{\rho}^{\prime}\right) \\
E_{v}(z, \boldsymbol{\rho})
\end{array}\right\}=4 \pi k_{0}^{2}\left\{\begin{array}{c}
0 \\
\delta\left(\mathbf{r}-\mathbf{r}^{\prime}\right) \delta_{\alpha \beta} \\
P_{\alpha}(z, \boldsymbol{\rho})
\end{array}\right\},
\end{aligned}
$$

где $\mathbf{r}=(z, \boldsymbol{\rho}), \boldsymbol{\rho}=(x, y), \quad k_{0}=\omega / c, c-$ скорость света. Строки (1)-(3) соответствуют отдельным уравнениям с одним и тем же оператором в левой части. Уравнение (1) определяет электрическое поле $\mathbf{E}^{(0)}$, а уравнение $(2)$ - тензорную функцию Грина $\hat{D}^{(0)}$ для невозмущенной среды с проницаемостью $\varepsilon^{(0)}(z, \omega)$, трансляционно-инвариантной по $\rho$. Решения уравнений (1) и (2) и их преобразования Фурье

$$
\begin{aligned}
& \left\{\mathbf{E}^{(0)}(z ; \boldsymbol{\kappa}), \hat{G}^{(0)}\left(z, z^{\prime} ; \boldsymbol{\kappa}\right)\right\} \\
& \quad=\int d^{2} \rho \exp (-i \boldsymbol{\kappa} \cdot \boldsymbol{\rho})\left\{\mathbf{E}^{(0)}(z, \boldsymbol{\rho}), \hat{D}^{(0)}\left(z, z^{\prime}, \boldsymbol{\rho}\right)\right\}
\end{aligned}
$$

должны удовлетворять максвелловским граничным условиям по переменной $z$. Поле $\mathbf{E}^{(0)}$ вычисляется далее при условии, что из оптически прозрачной среды 1 с проницаемостью $\varepsilon^{(0)}(z)=\varepsilon_{1}\left(\operatorname{Im} \varepsilon_{1}=0\right)$ вдоль оси $z$ $(\boldsymbol{\kappa}=0)$ падает монохроматическая волна

$$
\mathbf{E}^{\text {inc }}(z, \boldsymbol{\rho})=\mathbf{e}_{\alpha} \bar{E}^{\text {inc }} \exp \left(i \sqrt{\varepsilon_{1}} k_{0} z\right)
$$

линейно поляризованная вдоль орта $\mathbf{e}_{\alpha}$, где $\alpha$ обозначает $x$ или $y$. Функции $E_{\alpha}^{(0)}$ и $G_{\alpha \beta}^{(0)}$ в зависимости от $z$ и $z^{\prime}$ имеют макроскопический масштаб $\sim 1 / k_{0}$, как и поле (5), однородное в плоскостях $z=$ const.

Предположим, что в изолированной наночастице квазиоднородное световое поле $E_{\alpha} \mathbf{e}_{\alpha}$ наводит дипольный момент поверхностного плазмона

$$
\mathbf{p}=\chi_{\alpha \alpha}^{(0)}(\omega) E_{\alpha} \mathbf{e}_{\alpha}
$$

где $\chi_{\alpha \alpha}^{(0)}(\omega)$ - компонента тензора поляризуемости. Тогда в уравнении (3) поляризация, связанная с индуцированными диполями одинаковых частиц, имеет вид

$$
P_{\alpha}(z, \boldsymbol{\rho})=\delta\left(z-z_{0}\right) \sum_{\mathbf{n}} \chi_{\alpha \alpha}^{(0)}(\omega) \delta\left(\boldsymbol{\rho}-\boldsymbol{\rho}_{\mathbf{n}}\right) E_{\alpha}\left(z_{0}, \boldsymbol{\rho}_{\mathbf{n}}\right) .
$$

Наличие дельта-функции $\delta\left(z-z_{0}\right) \delta\left(\boldsymbol{\rho}-\boldsymbol{\rho}_{\mathbf{n}}\right)$ означает, что частицы малых размеров по сравнению с длиной волны света центрированы в точках $\mathbf{r}_{\mathbf{n}}=\left(z_{0}, \boldsymbol{\rho}_{\mathbf{n}}\right)$ плоскости $z=z_{0}$. Далее считаем, что они расположены в узлах прямоугольной решетки с векторами трансляции

$$
\boldsymbol{\rho}_{\mathbf{n}}=n_{x} A_{x} \mathbf{e}_{x}+n_{y} A_{y} \mathbf{e}_{y}
$$

где $A_{x}$ и $A_{y}$ - периоды решетки, $n_{x}$ и $n_{y}-$ номера ее узлов, $\mathbf{n}=\left(n_{x}, n_{y}\right)$. Предполагаем, что главные оси тензора поляризуемости $\hat{\chi}^{(0)}$ совпадают с ортами $\mathbf{e}_{\alpha}$ векторов решетки (8).

\section{3. Общее решение задачи}

В случае нормально падающей волны (5) уравнению (3) с поляризацией (7) эквивалентно следующее интегральное уравнение:

$$
\begin{aligned}
& E_{\alpha}(z, \boldsymbol{\rho})=E_{\alpha}^{(0)}(z \mid 0) \\
& \quad+\sum_{\beta} \int \frac{d^{2} \kappa}{(2 \pi)^{2}} G_{\alpha \beta}^{(0)}\left(z, z_{0} ; \boldsymbol{\kappa}\right) \chi_{\beta \beta}^{(0)} e^{i \boldsymbol{\kappa} \cdot \boldsymbol{\rho}} \tilde{E}_{\beta}\left(z_{0} ; \boldsymbol{\kappa}\right) .
\end{aligned}
$$

Здесь введено обозначение $E_{\alpha}^{(0)}(z \mid 0)=E_{\alpha}^{(0)}(z, \boldsymbol{\rho})$ для нормально распространяющейся невозмущенной волны с $\alpha=x$ или $y$ и представление

$$
\tilde{E}_{\beta}\left(z_{0} ; \boldsymbol{\kappa}\right)=\sum_{\mathbf{n}} e^{-i \boldsymbol{\kappa} \cdot \boldsymbol{\rho}_{\mathbf{n}}} E_{\beta}\left(z_{0}, \boldsymbol{\rho}_{\mathbf{n}}\right)
$$

для моды действующего (ближнего) поля $E_{\beta}\left(z_{0}, \boldsymbol{\rho}\right)$. Отметим различие обозначений $\mathbf{E}(z ; \boldsymbol{\kappa})$ для амплитуд Фурье вида (4), (10) и $\mathbf{E}(z \mid \boldsymbol{\kappa})$ для амплитуд волн $\mathbf{E}(z, \boldsymbol{\rho})=\mathbf{E}(z \mid \boldsymbol{\kappa}) \exp (i \boldsymbol{\kappa} \cdot \boldsymbol{\rho})$ в реальном пространстве.

Выполнив преобразование (10) выражения (9), получим уравнение для компонент $\tilde{E}_{\beta}\left(z_{0} ; \boldsymbol{\kappa}\right)=\tilde{E}_{\beta}\left(z_{0} ; \boldsymbol{\kappa}+\mathbf{b}_{\mathbf{m}}\right)$ ближнего поля (10) в квазистатическом приближении, когда $G_{\alpha \beta}^{(0)}=\delta_{\alpha \beta} G_{\alpha \alpha}^{(0)}$. При этом используем представление

$$
\sum_{\mathbf{n}} \exp \left(-i \boldsymbol{\kappa} \cdot \boldsymbol{\rho}_{\mathbf{n}}\right)=\frac{(2 \pi)^{2}}{A_{x} A_{y}} \sum_{\mathbf{m}} \delta\left(\boldsymbol{\kappa}-\mathbf{b}_{\mathbf{m}}\right),
$$

содержащее суммирование по номерам узлов $\mathbf{m}=\left(m_{x}, m_{y}\right)$ обратной решетки, или по ее векторам трансляции

$$
\mathbf{b}_{\mathbf{m}}=2 \pi\left(\frac{m_{x}}{A_{x}} \mathbf{e}_{x}+\frac{m_{y}}{A_{y}} \mathbf{e}_{y}\right)
$$

с целыми $m_{x}$ и $m_{y}$. 
Подставляя в (9) результаты самосогласованного решения уравнений для $\tilde{E}_{\beta}\left(z_{0} ; 0\right)$ с $\kappa=0$, для поля излучения находим

$$
\begin{aligned}
& E_{\beta}(z, \boldsymbol{\rho})=E_{\alpha}^{(0)}(z \mid 0) \delta_{\alpha \beta} \\
& \quad+\sum_{\mathbf{m}} e^{i \mathbf{b}_{\mathbf{m}} \cdot \boldsymbol{\rho}_{G_{\beta \alpha}}^{(0)}\left(z, z_{0} ; \mathbf{b}_{\mathbf{m}}\right) \frac{\chi_{\alpha \alpha}}{A_{x} A_{y}} E_{\alpha}^{(0)}\left(z_{0} \mid 0\right) .}
\end{aligned}
$$

Здесь $\chi_{\alpha \alpha}$ - эффективная плазмонная поляризуемость частицы в слое, которая соответствует представлению $p_{\alpha}=\chi_{\alpha \alpha} E_{\alpha}^{(0)} \quad$ дипольного момента (6) в формуле (7). Она получается при $\boldsymbol{\kappa}=0$ из общей формулы

$$
\chi_{\alpha \alpha}(\boldsymbol{\kappa})=\frac{\chi_{\alpha \alpha}^{(0)}}{1-\chi_{\alpha \alpha}^{(0)}\left(A_{x} A_{y}\right)^{-1} \sum_{\mathbf{m}} G_{\alpha \alpha}^{(0)}\left(z_{0}, z_{0} ; \boldsymbol{\kappa}+\mathbf{b}_{\mathbf{m}}\right)},
$$

найденной в квазистатическом приближении.

Выражение (12) описывает световое поле в слоистой среде с монослоем диполей, резонансно возбуждаемых волной (5). Поле (12) включает вклады c векторами обратной решетки $\mathbf{b}_{\mathbf{m} \neq 0}$ из (11), которые в случае короткопериодных решеток удовлетворяют условиям $\left|\mathbf{b}_{\mathbf{m}}\right| \gtrsim A_{\alpha}^{-1} \gg k_{0}$. Соответствующие компоненты поля, определяемые функциями $\left|G_{\beta \mu}^{(0)}\left(z, z_{0} ; \mathbf{b}_{\mathbf{m}}\right)\right| \propto \exp \left(-\left|\mathbf{b}_{\mathbf{m}}\right|\left|z-z_{0}\right|\right)$ в (12), затухают на субволновом расстоянии $\left|z-z_{0}\right| \sim A_{\alpha} \ll k_{0}^{-1}$, т.е. они отвечают за эффекты ближнеполевой оптики [15]. Поле излучения по нормали к поверхности $z=$ const в спектроскопии анизотропного отражения от короткопериодной решетки включает только вклад в (12) с $\mathbf{b}_{\mathbf{m}=0}=0$ и той же поляризацией $\alpha$, что у падающей волны (5).

Анизотропия компонент светового поля (12) при нормальном отражении обусловлена соотношением $\chi_{x x} \neq \chi_{y y}$. Оно определяется компонентами тензора $\hat{\chi}^{(0)}$ и квазистатическими решеточными суммами

$$
\frac{1}{A_{x} A_{y}} \sum_{\mathbf{m}} G_{\alpha \alpha}^{(0)}\left(z_{0}, z_{0} ; \mathbf{b}_{\mathbf{m}}\right)=\sum_{\mathbf{n}}^{\prime} D_{\alpha \alpha}^{(0)}\left(z_{0}, z_{0}, \boldsymbol{\rho}_{\mathbf{n}}\right)
$$

по узлам $\mathbf{m}$ обратной решетки или $\mathbf{n}$ прямой решетки. Далее мы изучим роль анизотропии этих двух типов по отдельности.

\section{4. Эффект локального поля в анизотропной наноструктуре}

Рассмотрим границу раздела $z=0$ двух сред, для чего полагаем в уравнениях $(1)-(3)$, что $\varepsilon^{(0)}(z)=\varepsilon_{1}$ при $z<0$ и $\varepsilon^{(0)}(z)=\varepsilon_{2}$ при $z>0$. Считаем, что в плоскости $z=z_{0}=-h<0$ вблизи границы раздела сред расположен слой диполей. Поле $E_{\alpha}=E_{\alpha}^{(0)}+E_{\alpha}^{(d)}+E_{\alpha}^{(i)}$, действующее в решетке (8) на диполь в выделенном узле $\mathbf{r}_{\mathbf{n}=0}=\left(z_{0}, \boldsymbol{\rho}_{\mathbf{n}}=0\right)$, включает невозмущенное по- ле $E_{\alpha}^{(0)}$ и поля

$$
\left.\begin{array}{c}
E_{\alpha}^{(d)}=S_{\alpha}^{(d)} \frac{\chi_{\alpha \alpha}^{(0)}}{\varepsilon_{1} A_{\alpha}^{3}} E_{\alpha}, \\
E_{\alpha}^{(i)}=S_{\alpha}^{(i)}(h) \frac{\chi_{\alpha \alpha}^{(0)}}{\varepsilon_{1} A_{\alpha}^{3}} \frac{\varepsilon_{1}-\varepsilon_{2}}{\varepsilon_{1}+\varepsilon_{2}} E_{\alpha} .
\end{array}\right\}
$$

Величины $S_{\alpha}^{(d)}$ и $S_{\alpha}^{(i)}$ получаются в пренебрежении запаздыванием из сумм $\sum_{\mathbf{n}}^{\prime} D_{\alpha \alpha}^{(0)}\left(z_{0}, z_{0}, \boldsymbol{\rho}_{\mathbf{n}}\right)$, входящих в $\chi_{\alpha \alpha}$ согласно $(13),(14)$. Поле $\mathbf{E}^{(d)}$ создается диполями, находящимися в узлах решетки с $\boldsymbol{\rho}_{\mathbf{n}} \neq 0$, а поле $\mathbf{E}^{(i)}-$ решеткой диполей изображения, возникающих на границе раздела сред $\varepsilon_{1} \mid \varepsilon_{2}$. С учетом поправок на локальное поле (15) и представлений $p_{\alpha}=\chi_{\alpha \alpha}^{(0)} E_{\alpha}=\chi_{\alpha \alpha} E_{\alpha}^{(0)}$ для дипольного момента (6) выражение для компонент тензора эффективной поляризуемости принимает вид

$$
\chi_{\alpha \alpha}=\left[\frac{1}{\chi_{\alpha \alpha}^{(0)}}-\frac{1}{\varepsilon_{1} A_{\alpha}^{3}}\left(S_{\alpha}^{(d)}+\frac{\varepsilon_{1}-\varepsilon_{2}}{\varepsilon_{1}+\varepsilon_{2}} S_{\alpha}^{(i)}\right)\right]^{-1} .
$$

Эти компоненты описывают длинноволновый $(\boldsymbol{\kappa}=0)$ отклик на макроскопическое поле $E^{(0)}$, которое определяется из уравнения (1).

В выражения (15) и (16) входит решеточная сумма

$$
\begin{aligned}
S_{\alpha}^{(d)} & =A_{\alpha}^{3} \sum_{n_{\alpha}, n_{\beta}=-\infty}^{\infty} \frac{3 \rho_{\mathbf{n}, \alpha}^{2}-\rho_{\mathbf{n}}^{2}}{\rho_{\mathbf{n}}^{5}} \\
& =4\left[\zeta(3)-8 \pi^{2} \sum_{m_{\alpha}=1}^{\infty} \sum_{m_{\beta}=1}^{\infty} m_{\alpha}^{2} K_{0}\left(2 \pi m_{\alpha} m_{\beta} \frac{A_{\beta}}{A_{\alpha}}\right)\right],
\end{aligned}
$$

в которой индексы $\alpha$ и $\beta$ равны $x$ и $y$, причем если $\alpha=x$, то $\beta=y$, и наоборот. Первое равенство в (17) относится к суммированию по узлам прямоугольной решетки (8) с $\boldsymbol{\rho}_{\mathbf{n}} \neq 0$. Быстро сходящийся ряд по узлам обратной решетки (11) дается вторым равенством в (17), где $\xi(p)-$ дзета-функция Римана, $K_{0}(z)-$ функция Макдональда. Функции $S_{\alpha}^{(d)}$, рассчитанные численно в зависимости от отношения $A_{x} / A_{y}$, приведены в работе [3].

Решеточная сумма, которая связана с диполями изображения, находящимися в узлах решетки $\mathbf{r}_{\mathbf{n}}^{\prime}=\left(h, \boldsymbol{\rho}_{\mathbf{n}}\right)$ на расстоянии $h$ от границы раздела сред, равна

$$
\begin{aligned}
S_{\alpha}^{(i)}(h)= & -\left(\frac{A_{\alpha}}{2 h}\right)^{3}+A_{\alpha}^{3} \sum_{n_{\alpha}, n_{\beta}=-\infty}^{\infty} \frac{3 \rho_{\mathbf{n}, \alpha}^{2}-\left(\rho_{\mathbf{n}}^{2}+4 h^{2}\right)}{\left(\rho_{\mathbf{n}}^{2}+4 h^{2}\right)^{5 / 2}} \\
= & -8 \pi^{2} \sum_{m_{\alpha}=1}^{\infty} \sum_{m_{\beta}=-\infty}^{\infty} \frac{m_{\alpha}^{2}}{\sqrt{m_{\alpha}^{2}\left(A_{\beta} / A_{\alpha}\right)^{2}+m_{\beta}^{2}}} \\
& \times \exp \left(-\frac{4 \pi h}{A_{\beta}} \sqrt{m_{\alpha}^{2}\left(A_{\beta} / A_{\alpha}\right)^{2}+m_{\beta}^{2}}\right) .
\end{aligned}
$$

Здесь индексы $\alpha$ и $\beta$ имеют тот же смысл, что в выражении (17). В первом соотношении (18) выделен 
расходящийся при $h \rightarrow 0$ член, который соответствует вкладу собственного изображения выделенного диполя [16].

\section{5. Наблюдаемые величины}

В случае падающей волны (5) поле излучения по нормали в область $z \rightarrow-\infty$

$$
E_{\alpha}(z, \boldsymbol{\rho})-E_{\alpha}^{\text {inc }}(z, \boldsymbol{\rho})=\left(r^{(0)}+\Delta r_{\alpha}\right) \bar{E}^{\mathrm{inc}} \exp \left(-i \sqrt{\varepsilon_{1}} k_{0} z\right)
$$

получается из (12) при учете только члена $\mathbf{c} \mathbf{b}_{\mathbf{m}=0}=0$ в сумме. Коэффициент отражения света $r_{\alpha}=r^{(0)}+\Delta r_{\alpha}$ в среду с $\varepsilon^{(0)}(z)=\varepsilon_{1}$ включает вклады

$$
r^{(0)}=\frac{\sqrt{\varepsilon_{1}}-\sqrt{\varepsilon_{2}}}{\sqrt{\varepsilon_{1}}+\sqrt{\varepsilon_{2}}}
$$

плоской границы раздела $\varepsilon_{1} \mid \varepsilon_{2}$ и

$$
\Delta r_{\alpha}=\frac{2 \pi i k_{0}}{\sqrt{\varepsilon_{1}}} \frac{\chi_{\alpha \alpha}}{A_{x} A_{y}}\left(e^{-i \sqrt{\varepsilon_{1}} k_{0} h}+r^{(0)} e^{i \sqrt{\varepsilon_{1}} k_{0} h}\right)^{2}
$$

слоя частиц с эффективными поляризуемостями $\chi_{\alpha \alpha}$.

Методом спектроскопии анизотропного отражения света регистрируется сигнал $[5,6]$

$$
\frac{\Delta R}{R}=2 \frac{R_{x}-R_{y}}{R_{x}+R_{y}}
$$

в зависимости от энергии фотонов. В формуле (22) $R_{\alpha}=\left|r_{\alpha}\right|^{2}$ - коэффициент нормального отражения света с поляризацией по оси $\alpha$. В случае резонансного отражения спектр (22) удобно вычислять на основе представления

$$
\frac{k_{0}}{A_{x} A_{y}} \chi_{\alpha \alpha}(\omega) \approx \frac{\Omega_{\alpha}^{2}}{\omega_{\alpha}^{2}-\omega^{2}-i \omega \Gamma_{\alpha}} .
$$

Феноменологически оно соответствует модели лоренцевых осцилляторов для анизотропных плазмонов. При условиях $\left|\Delta r_{\alpha}\right| \ll\left|r^{(0)}\right|$ и $\sqrt{\varepsilon_{1}} k_{0} h \ll 1$ выражение (22) принимает простой вид

$$
\frac{\Delta R}{R}=\frac{16 \pi \sqrt{\varepsilon_{1}} k_{0}}{A_{x} A_{y}} \operatorname{Im}\left(\frac{\chi_{y y}-\chi_{x x}}{\varepsilon_{1}-\varepsilon_{2}}\right) .
$$

В случае эффективных поляризуемостей (23) выражение (24) описывает резонансный спектр $\Delta R / R$.

\section{6. Анизотропия формы частиц и структуры решетки}

Изучим эффекты анизотропии в спектре отражения (20)-(22), используя модель одинаковых наночастиц эллипсоидальной формы. Компоненты тензора поляризуемости изолированной частицы имеют вид [17]

$$
\chi_{\alpha \alpha}^{(0)}=\frac{a_{x} a_{y} a_{z}}{3} \frac{\varepsilon-\varepsilon_{1}}{\left(\varepsilon-\varepsilon_{1}\right) N^{(\alpha)}+\varepsilon_{1}} .
$$

Здесь $\varepsilon$ и $\varepsilon_{1}$ - проницаемости материала частицы и однородной окружающей среды. Коэффициенты деполяризации эллипсоида [17]

$$
N^{(\alpha)}=\frac{a_{x} a_{y} a_{z}}{2} \int_{0}^{\infty} \frac{d s}{\sqrt{\left(s+a_{\alpha}^{2}\right)^{3}\left(s+a_{\beta}^{2}\right)\left(s+a_{\gamma}^{2}\right)}},
$$

где $\alpha \neq \beta \neq \gamma \neq \alpha$, зависят от длин $a_{x}, a_{y}, a_{z}$ его полуосей и удовлетворяют условию $N^{(x)}+N^{(y)}+N^{(z)}=1$.

Далее будем считать, что наночастицы образованы металлом с диэлектрической проницаемостью

$$
\varepsilon(\omega)=\varepsilon_{\infty}-\frac{\omega_{p}^{2}}{\omega^{2}+i \omega \gamma},
$$

где $\omega_{p}-$ плазменная частота, $1 / \gamma-$ время релаксации электронов. Из (25) следует уравнение $\left(\operatorname{Re} \varepsilon(\omega)-\varepsilon_{1}\right) N^{(\alpha)}+\varepsilon_{1}=0$, которое при учете $(27)$ дает частоту

$$
\omega_{\alpha}^{(0)}=\omega_{p} \sqrt{N^{(\alpha)} / \varepsilon_{*}^{(\alpha)}}
$$

дипольного плазмона, поляризованного вдоль $\alpha$-й полуоси эллипсоида. Частота (28) с $\varepsilon_{*}^{(\alpha)}=\left(\varepsilon_{\infty}-\varepsilon_{1}\right) N^{(\alpha)}$ $+\varepsilon_{1}$ найдена с точностью до членов $\sim\left(\gamma / \omega_{\alpha}^{(0)}\right)^{2} \ll 1$; она зависит от длин полуосей эллипсоида через $N^{(\alpha)}$.

Определим параметры эффективной поляризуемости (23) ab initio в модели слоя наночастиц, для чего подставим (25) в (16). Преобразование последнего выражения к виду (23) дает для длинноволновой $(\boldsymbol{\kappa}=0)$ плазмонной моды с поляризацией $\alpha$ в плоскости слоя значения

$$
\begin{aligned}
\omega_{\alpha}=\omega_{\alpha}^{(0)} & \left\{1-\frac{a_{x} a_{y} a_{z}}{3 A_{\alpha}^{3}} \frac{1}{\varepsilon_{*}^{(\alpha)} N^{(\alpha)}}\right. \\
\times & {\left.\left[S_{\alpha}^{(d)}+S_{\alpha}^{(i)} \mu^{\prime}+\frac{\gamma}{\omega_{\alpha}^{(0)}} \frac{\varepsilon_{\infty}-\varepsilon_{1}}{\varepsilon_{1}} N^{(\alpha)} S_{\alpha}^{(i)} \mu^{\prime \prime}\right]\right\}^{1 / 2}, } \\
\Gamma_{\alpha}= & \gamma+\frac{a_{x} a_{y} a_{z}}{3 A_{\alpha}^{3}} \omega_{\alpha}^{(0)} \frac{1}{\varepsilon_{*}^{(\alpha)} N^{(\alpha)}} \\
& \times\left[S_{\alpha}^{(i)} \mu^{\prime \prime}-\frac{\gamma}{\omega_{\alpha}^{(0)}} \frac{\varepsilon_{\infty}-\varepsilon_{1}}{\varepsilon_{1}} N^{(\alpha)}\left(S_{\alpha}^{(d)}+S_{\alpha}^{(i)} \mu^{\prime}\right)\right] \\
& \Omega_{\alpha}^{2}=\frac{\omega_{\alpha}}{c A_{x} A_{y}} \frac{a_{x} a_{y} a_{z}}{3}\left(\omega_{\alpha}^{(0)}\right)^{2} \frac{\varepsilon_{1}}{\varepsilon_{*}^{(\alpha)} N^{(\alpha)}}
\end{aligned}
$$

Здесь $S_{\alpha}^{(i)}<0$ и

$$
\mu=\frac{\varepsilon_{1}-\varepsilon_{2}}{\varepsilon_{1}+\varepsilon_{2}}=\frac{\varepsilon_{1}^{2}-\left|\varepsilon_{2}\right|^{2}-2 i \varepsilon_{1} \varepsilon_{2}^{\prime \prime}}{\left(\varepsilon_{1}+\varepsilon_{2}^{\prime}\right)^{2}+\left(\varepsilon_{2}^{\prime \prime}\right)^{2}},
$$

причем $\mu^{\prime}=\operatorname{Re} \mu<0$ и $\mu^{\prime \prime}=\operatorname{Im} \mu<0$ при $1 \stackrel{\sim}{<} \varepsilon_{1}<\left|\varepsilon_{2}\right|$. Далее мы свяжем наблюдаемую анизотропию плазмонных частот с анизотропией геометрических характеристик массива частиц. Экспериментальные спектры анизотропного отражения $\Delta R / R \quad[6]$ указывают на слабую анизотропию плазмонных частот 
$\left|\omega_{x}-\omega_{y}\right| \ll \omega_{x}, \omega_{y}$. В общем случае формула (29) учитывает два источника анизотропии плазмонных частот $\omega_{x} \neq \omega_{y}$. Ими являются (I) анизотропия формы частиц с $a_{x} \neq a_{y}$ при $A_{x}=A_{y}=A$ (квадратная решетка) и (II) анизотропия структуры слоя с $A_{x} \neq A_{y}$ при $a_{x}=a_{y}=a$ (сфероиды). В случае II условие $\omega_{x} \neq \omega_{y}$ связано с анизотропией дипольных взаимодействий между плазмонами разных частиц.

В изотропной модели, для которой $\Delta R / R=0$, введем плазмонную частоту $\omega_{\|}$, соответствующую наличию вырождения частот $\omega_{\alpha}$ с $\alpha=x, y$ из (29) по поляризации в плоскости $x y$. Частоту $\omega_{\|}$и ее малое анизотропное расщепление $\Delta \omega_{\|}$выразим формулами

$$
\omega_{\|}=\frac{1}{2}\left(\omega_{x}+\omega_{y}\right), \quad \Delta \omega_{\|}=\omega_{x}-\omega_{y} .
$$

В случае (I) расщепление $\Delta \omega_{\|} \neq 0$ связываем с анизотропией длин полуосей $a_{x, y}=a \mp \Delta a / 2,|\Delta a| \ll a$ эллипсоидов, заполняющих квадратную решетку. В случае (II) расщепление $\Delta \omega_{\|} \neq 0$ связываем с анизотропией периодов $A_{x, y},=A \mp \Delta A / 2,|\Delta A| \ll A$ решетки сфероидов. Разложение функций $\omega_{\alpha}\left(a_{\alpha}, A_{\alpha}\right)$ из (29) в линейном приближении по $\Delta a$ и $\Delta A$ вблизи значений параметров $a, A$ дает

$$
\frac{\omega_{x}-\omega_{y}}{\omega_{\|}}=C_{\mathrm{I}} \frac{\Delta a}{a}+C_{\mathrm{II}} \frac{\Delta A}{A} .
$$

Вычислим коэффициенты $C_{\mathrm{I}}$ и $C_{\mathrm{II}}$, отмечая индексом \| величины, относящиеся к изотропной модели с параметрами $a, A$. Анизотропия формы частиц в квадратной решетке связана с деформацией кругового сечения сфероидов в эллиптическое. Разлагая выражение $N^{(\alpha)}=N^{\|}+\Delta N^{(\alpha)}$ из (26) по $\Delta a$, получаем $\Delta N^{(x)}=-\Delta N^{(y)}=\Delta N^{\|}$. При этом в формуле (34) находим

$$
\begin{aligned}
C_{\mathrm{I}}= & -\frac{1}{4}\left[\frac{\varepsilon_{1}}{\varepsilon_{*}^{\|}}+\frac{U}{1-U}\left(1+\frac{\varepsilon_{\infty}-\varepsilon_{1}}{\varepsilon_{*}^{\|}} N^{\|}\right)\right] \\
& \times \frac{1}{1-\eta^{2}} \frac{\left(5-2 \eta^{2}\right) \eta^{2} f-3 \operatorname{arctg} f}{\operatorname{arctg} f-\eta^{2} f}
\end{aligned}
$$

где

$$
U=\frac{\eta}{3}\left(\frac{a}{A}\right)^{3} \frac{1}{\varepsilon_{*}^{\|} N^{\|}}\left(S_{\|}^{(d)}+S_{\|}^{(i)} \operatorname{Re} \mu\right),
$$

$\varepsilon_{*}^{\|}=\left(\varepsilon_{\infty}-\varepsilon_{1}\right) N^{\|}+\varepsilon_{1}, \quad \eta=a_{z} / a, \quad f=\sqrt{1-\eta^{2}} / \eta=$ $\sqrt{\left(a / a_{z}\right)^{2}-1}, \quad S_{\|}^{(d)} \quad$ и $S_{\|}^{(i)}-$ суммы $(17)$ и $(18)$, вычисленные для квадратной решетки с периодом $A$. Величина $\mu$ дается формулой (32), вклад с $\mu^{\prime \prime}$ из выражения (36) исключен вследствие малости параметра $\left(\gamma / \omega_{\|}^{(0)}\right)\left|\varepsilon_{\infty} / \varepsilon_{1}-1\right| \ll 1$ в $(29)$.

Анизотропия структуры массива частиц определяется прямоугольной решеткой с $A_{x, y}=A \mp \Delta A / 2$. При этом из разложения (29) по $\Delta A$ получаем

$$
C_{\mathrm{II}}=-\frac{3}{2} \frac{U}{1-U} \frac{\sigma_{\|}^{(d)}+\sigma_{\|}^{(i)} \operatorname{Re} \mu}{S_{\|}^{(d)}+S_{\|}^{(i)} \operatorname{Re} \mu} .
$$

Здесь стоит $U$ из формулы (36), суммы

$$
\left.\begin{array}{c}
\sigma_{\|}^{(d)}=\sum_{n, m=-\infty}^{\infty} n^{2} \frac{3 n^{2}-7 m^{2}}{\left(n^{2}+m^{2}\right)^{7 / 2}}, \\
\sigma_{\|}^{(i)}=\sum_{n, m=-\infty}^{\infty} n^{2} \frac{3 n^{2}-7 m^{2}-2(2 h / A)^{2}}{\left(n^{2}+m^{2}+(2 h / A)^{2}\right)^{7 / 2}}
\end{array}\right\}
$$

выражаются через производные по $A_{x}$ и $A_{y}$ сумм (17) и (18), взятые при $A_{\alpha}=A$, а штрих означает отсутствие в сумме члена с $n=m=0$.

Заметим, что, хотя мы использовали модель периодического массива наночастиц, результаты теории качественно применимы к длинноволновым плазмонам в апериодических макроскопически однородных массивах [5,6]. Действительно, в них когерентность между дипольными плазмонами обеспечивается квазиоднородным возбуждающим полем, при этом роль мелкомасштабного беспорядка становится незначительной [18]. Вследствие гомогенизации отклика в разупорядоченных средах с дальнодействием существуют длинноволновые поляризационные возбуждения с качественно такими же свойствами, как в соответствующих кристаллах. Для примера укажем наличие поверхностных плазмонов в жидких металлах [19], поверхностных фононных поляритонов [20] и продольно-поперечного расщепления фононов [21] в полярных стеклах, экситонных поляритонов в полупроводниковых твердых растворах [22] и т.д. Эти представления распространяются и на длинноволновые моды дипольных плазмонов в разупорядоченных массивах частиц.

\section{7. Численный анализ спектров и оценка анизотропии}

Перейдем к интерпретации наблюдаемых спектров $\Delta R / R$ в рамках представленной выше теории и оценке степени анизотропии массивов наночастиц. Вначале рассматривая функцию вида (23) как феноменологическую, получим значения ее параметров путем непосредственной подгонки спектров $\Delta R / R$ из (20)-(22) под эксперимент [6]. Затем выполним $a b$ initio расчет плазмонных спектров $\Delta R / R$ с теоретической функцией (23), выраженной через оптические характеристики (29)-(31) плазмонов. Наконец, используя зависимость величин (29)-(31) от геометрических параметров модели, оценим степень анизотропии массивов частиц, ответственных за наблюдаемые спектры $\Delta R / R$.

7.1. Подгонка спектров. Спектры анизотропного отражения $\Delta R / R$, наблюдавшиеся для массивов металлических нанокластеров [4-6], показывают наличие характерной структуры в области плазмонных резонансов. Как пример, на рис. $1, a, c$ и $e$ точками изображены экспериментальные спектры $\Delta R / R$ нанокластеров индия, сформированных на разных участках поверхности (001) 

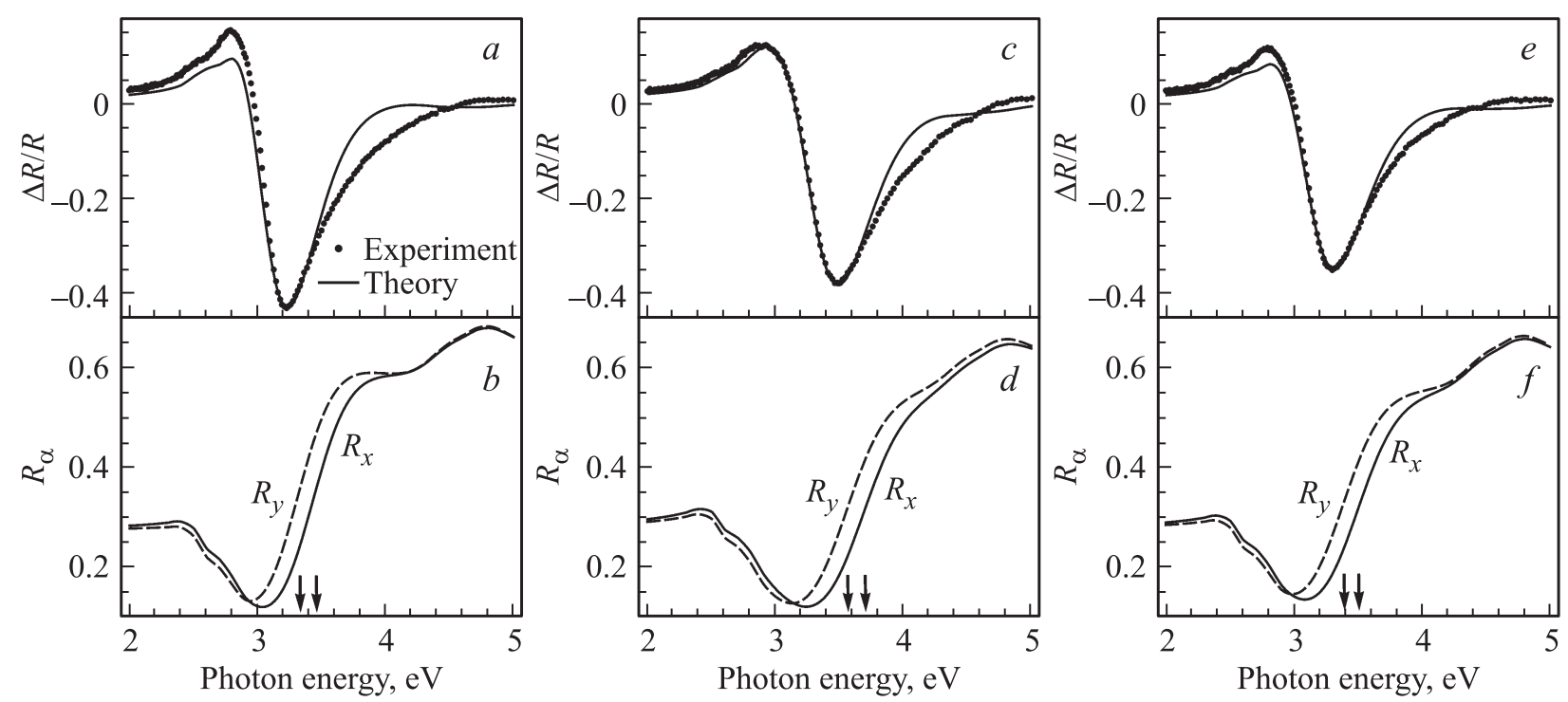

Рис. 1. $a, c, e-$ спектры анизотропного отражения света $\Delta R / R$ (точки), измеренные [6] в разных частях массива нанокластеров индия на поверхности (001) InAs, и результаты подгонки по формулам (20)-(22) (сплошные линии). $b, d, f-$ соответствующие спектры поляризованного отражения $R_{y}$ и $R_{x}$, вычисленные при подгонке. Энергии плазмонов $\hbar \omega_{y}$ и $\hbar \omega_{x}$ в спектрах $R_{y}$ и $R_{x}$ показаны левой и правой стрелками соответственно. Полученные параметры феноменологической функции вида (23) представлены в таблице.

InAs [6]. Сплошными линиями показаны результаты подгонки спектров $\Delta R / R$ при использовании для плазмонов феноменологической поляризуемости (23). В таблице приведены параметры функций (23), найденные в предположении, что $\omega_{x} \neq \omega_{y}$, а величины $\Gamma$ и $\Omega$ одинаковы для осцилляторов с $\alpha=x$ и $y$.

Для подгонки спектров $\Delta R / R$ использовалась формула (22) после подстановки в нее спектров отражения $R_{\alpha}=\left|r^{(0)}+\Delta r_{\alpha}\right|^{2}$ из выражений (20) и (21). Последние при $\Delta r_{\alpha}=0$ дают коэффициент отражения света $\left|r^{(0)}\right|^{2}$ от границы раздела сред $\varepsilon_{1} \mid \varepsilon_{2}$ в отсутствие частиц. При $r^{(0)}=0$ они выражают коэффициенты анизотропного отражения $\left|\Delta r_{\alpha}\right|^{2}$ слоем частиц, находящихся в однородной среде $\left(\varepsilon_{1}=\varepsilon_{2}\right)$. По отдельности спектры $\left|r^{(0)}\right|^{2}$ и $\left|\Delta r_{\alpha}\right|^{2} \sim\left|\chi_{\alpha \alpha}^{(0)}\right|^{2}$ имеют простую форму, в частности последний, согласно (23), имеет резонансный характер в области плазмонных частот. Однако форма спектров $R_{\alpha}=\left|r^{(0)}+\Delta r_{\alpha}\right|^{2}$ может быть многообразной в зависимости от фаз интерферирующих амплитуд $r^{(0)}$ и $\Delta r_{\alpha}$. Это видно из спектров $R_{\alpha}$, представленных на рис. $1, b, d$ и $f$ в качестве промежуточного результата при подгонке спектров $\Delta R / R$. При расчете спектров $\Delta R / R$ требуется выполнение двух физических условий: 1) должно быть $\left|\Delta r_{\alpha}\right|^{2}<1, R_{\alpha}<1$, и 2) сумма (18), расходящаяся при $h \rightarrow 0$, не должна превышать сумму (17).

Согласие между результатами подгонки и экспериментальными спектрами $\Delta R / R$ на рис. $1, a, c$ и $e$ вполне удовлетворительное. Следует подчеркнуть, что найденные частоты минимумов в спектрах $\Delta R / R$ не совпадают с частотами плазмонов $\omega_{\alpha}$, которые показаны стрелками на рис. $1, b, d$ и $f$. Разности частот $\omega_{x}-\omega_{y}$, являющиеся мерой анизотропии слоя частиц, малы по сравнению с частотами $\omega_{\alpha}$ и ширинами $Г$ плазмонных резонансов.

7.2. Модельный анализ параметров анизотропии. Проинтерпретируем теперь результаты подгонки спектров $\Delta R / R$ (см. таблицу), выполнив их теоретический анализ в модели слоя эллипсоидов. Начнем с оценки плазмонных частот в изотропной модели с $a_{\alpha}=a$ и $A_{\alpha}=A$. Частота плазмона в изолированном сфероиде

Значения параметров в феноменологических выражениях вида (23), полученные в результате подгонки теоретических спектров $\Delta R / R$ (непрерывные линии на рис. $1, a, c, e)$ под экспериментальные (точки на рис. $1, a, c, e[6])$. При расчете по формулам (20)-(22) считалось, что $\omega_{x} \neq \omega_{y}$, а $\Gamma$ и $\Omega$ одинаковы для компонент $\chi_{\alpha \alpha}$ с поляризациями $\alpha=x, y$.

\begin{tabular}{c|c|c|c|c|c|c}
\hline $\begin{array}{c}\text { Рисунок, } \\
\text { представляющий } \\
\text { спектр } \Delta R / R\end{array}$ & $\hbar \omega_{x}, \mathrm{eV}$ & $\hbar \omega_{y}, \mathrm{eV}$ & $\hbar \omega_{\|}, \mathrm{eV}$ & $\hbar \Delta \omega_{\|}, \mathrm{eV}$ & $\hbar \Gamma, \mathrm{eV}$ & $\hbar \Omega, \mathrm{eV}$ \\
\hline $1, a$ & 3.46 & 3.34 & 3.40 & 0.12 & 1.07 & 0.78 \\
$1, c$ & 3.71 & 3.58 & 3.645 & 0.13 & 1.17 & 0.78 \\
$1, e$ & 3.52 & 3.40 & 3.46 & 0.12 & 1.17 & 0.77
\end{tabular}



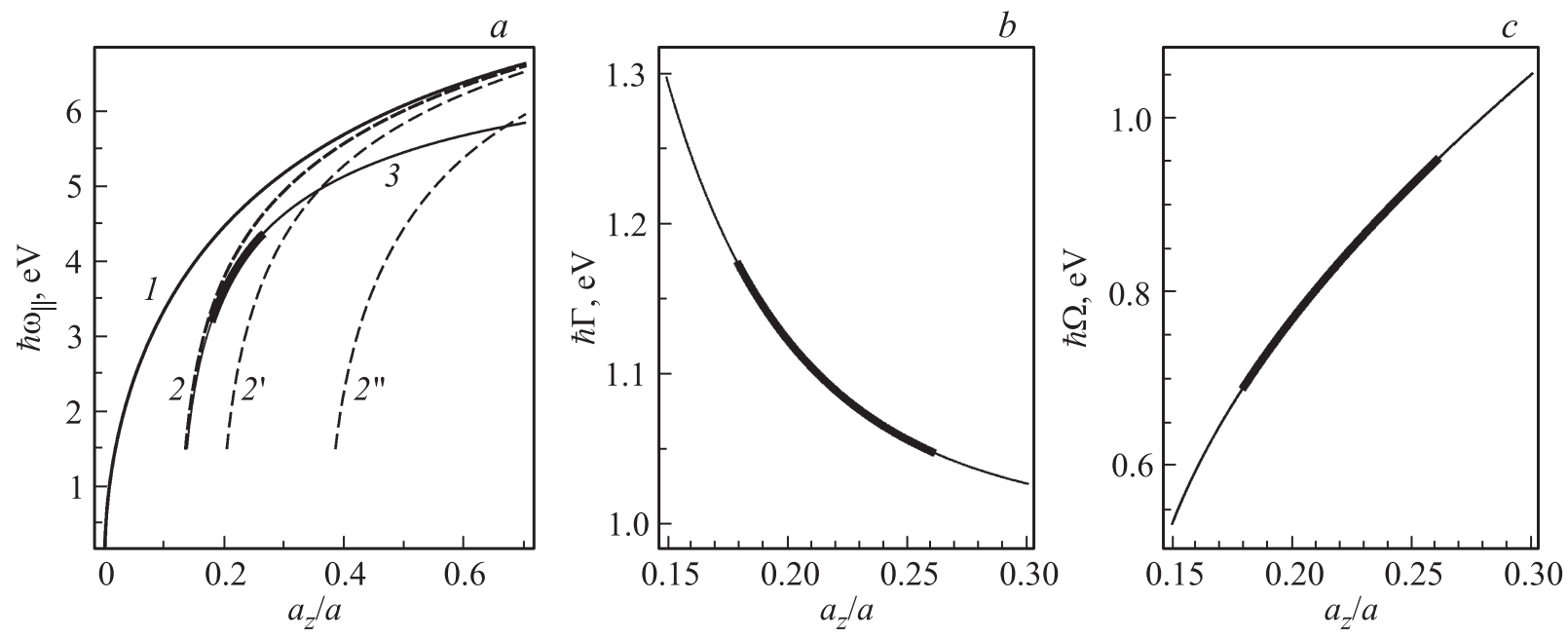

Рис. 2. $a$ - вычисленные в зависимости от $\eta=a_{z} / a$ частоты плазмонов: $\omega_{\|}^{(0)}$ из выражения (28) для изолированного сфероида (1), $\omega_{\|}^{(s)}$ из (39) для одиночного сфероида с учетом сил изображения при $p_{h}=3(2), p_{h}=2\left(2^{\prime}\right), p_{h}=1.1\left(2^{\prime \prime}\right), \omega_{\|}$из (29) для слоя сфероидов с учетом межчастичного взаимодейстия и сил изображения при $p_{h}=3(3)$. $b-$ параметр затухания (30), $c-$ „сила осциллятора“ (31) плазмонных мод в слое сфероидов. Для выделенных жирным отрезков кривых на панелях $a-c$ выполнено условие $R_{\alpha}<1$. Вычислено при $a=20 \mathrm{~nm}, A=50 \mathrm{~nm}$ с диэлектрическими проницаемостями In из [23] и InAs из [24]. Энергии $\hbar \omega=3.45 \mathrm{eV}$ на кривой 3 соответствует $\eta \approx 0.2\left(a_{z}=4 \mathrm{~nm}\right)$.

равна $\omega_{\|}^{(0)}$ из (28), а с учетом сил изображения для того же сфероида, расположенного на расстоянии $h$ от поверхности, она преобразуется в частоту

$$
\omega_{\|}^{(s)}=\omega_{\|}^{(0)}\left(1+\frac{\eta}{24}\left(\frac{a}{h}\right)^{3} \frac{1}{N^{\|} \mathcal{E}_{*}^{\|}} \operatorname{Re} \mu\right)^{1 / 2} .
$$

Длинноволновая плазмонная мода массива частиц вблизи поверхности имеет частоту $\omega_{\|}$из (29), в которой учтено межчастичное дипольное взаимодейстие. На рис. 2, $a$ приведены зависимости частот $\omega_{\|}^{(0)}$ (кривая 1$), \omega_{\|}^{(s)}$ (кривые 2,2', $2^{\prime \prime}$ ) и $\omega_{\|}$(кривая 3) от $\eta=a_{z} / a$ для наночастиц индия с $a=20 \mathrm{~nm}$ на поверхности InAs. Значения масштабирующих параметров $p_{A}=A / a$ и $p_{h}=h /(\eta a)$ равны $p_{A}=2.5$ и $p_{h}=3$. Из рис. 2, $a$ видно, что при уменьшении $\eta$ плазмонные частоты испытывают поляризационное „смягчение“ по сравнению с $\omega_{\|}^{(0)}$, причем $\omega_{\|}^{(s)} \rightarrow+0$ и $\omega_{\|} \rightarrow+0$ при критических значениях $\eta$, которые зависят от $p_{h}$ и $p_{A} / p_{h}$. В этом состоит проявление хорошо известного эффекта сил изображения для точечных резонансных диполей [16].

Чтобы найти физически обоснованное значение $\eta$, в соответствии с формулой (33) отождествим теоретическую частоту $\omega_{\|}$для изотропной модели с экспериментальной величиной $\left(\omega_{x}+\omega_{y}\right) / 2$ из таблицы. В случае $\hbar \omega_{\|}=3.45 \mathrm{eV}$ рис. $2, a$ дает значение $\eta \approx 0.2$, которое обеспечивает выполнение условий $R_{\alpha}<1$. При $\eta \approx 0.2$ из рис. $2, b$ и $2, c$ находим теоретические значения $\hbar \Gamma=1.12 \mathrm{eV}$ и $\hbar \Omega=0.77 \mathrm{eV}$, близкие к результам подгонки из таблицы. Подставим эти значения $\Gamma$ и $\Omega$,

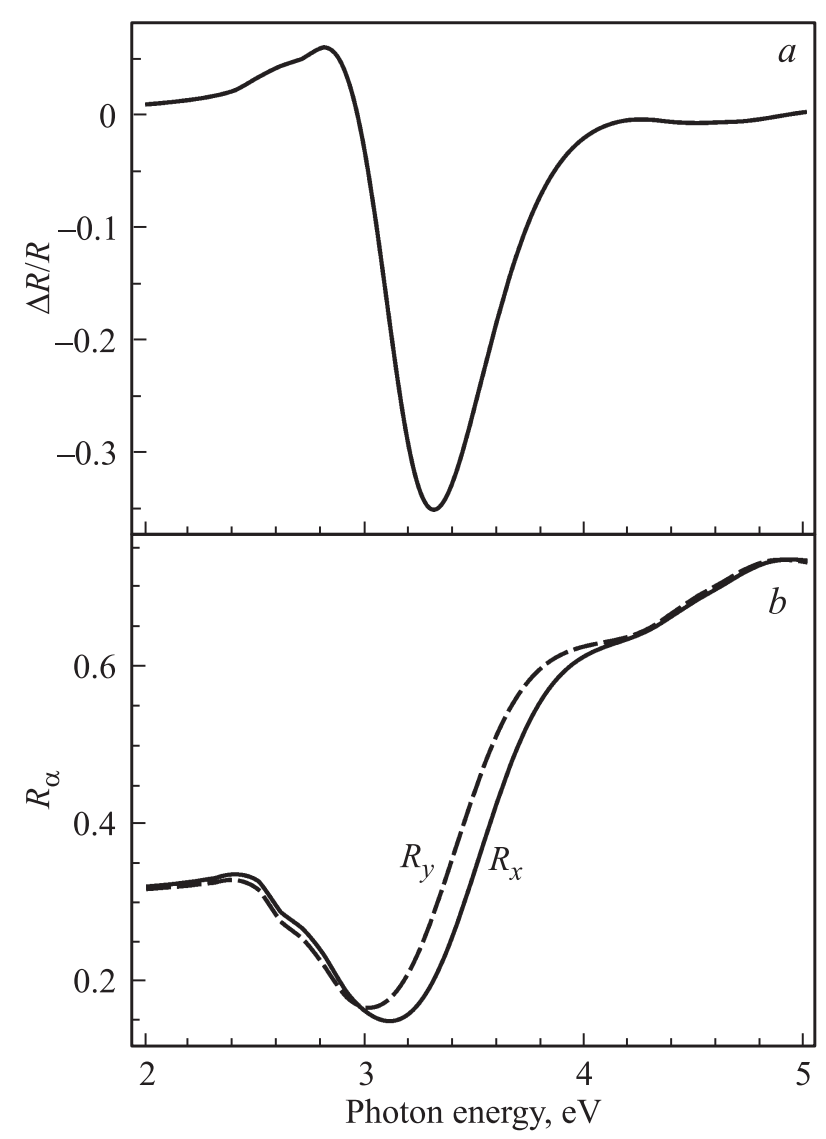

Рис. 3. Теоретические спектры $\Delta R / R(a), R_{y}$ и $R_{x}(b)$, вычисленные для модели прямоугольной решетки эллипсоидов In/InAs. Использованы те же значения параметров, что на puc. 2: $a=20 \mathrm{~nm}, A=50 \mathrm{~nm}, p_{h}=3, a_{z}=4 \mathrm{~nm}(\eta \approx 0.2)$, $h=12 \mathrm{~nm}$. Величина $\hbar \Delta \omega_{\|}=0.12 \mathrm{eV}$ взята в соответствии с таблицей. 
а также $\hbar \omega_{x, y}=3.45 \pm 0.06 \mathrm{eV}$ в $\chi_{\alpha \alpha}$ из (23), результаты - в формулы (20)-(22). Этот расчет ab initio дает представленные на рис. 3 теоретические спектры $\Delta R / R$ и $R_{\alpha}$ в хорошем соответствии с результатами эксперимента и подгонки (рис. 1). Полученные теоретические результаты соответствуют геометрическим параметрам модели $a=20 \mathrm{~nm}, a_{z}=4 \mathrm{~nm}, h=12 \mathrm{~nm}$, $A=50 \mathrm{~nm}$.

В представленной теории величины $\Delta a / a$ и $\Delta A / A$ являются мерой макроскопической анизотропии слоя металлических нанокластеров. Оценим на основе формулы (34) величины $\Delta a / a$ и $\Delta A / A$, соответствующие заданному (наблюдаемому) расщеплению частот $\Delta \omega_{\|} / \omega_{\|}$. Из формул (35) и (36) при найденных выше значениях параметров модели получаем $C_{\mathrm{I}}=1.12$. Подстановка его в (34) показывает, что величине $\Delta \omega_{\|} / \omega_{\|}=0.12 / 3.45$ соответствует деформация сфероидов $\Delta a / a \approx a_{y} / a_{x}-1 \approx 3.1 \%$, если $\Delta A=0$. Аналогично из выражений (37) и (38) следует $C_{\text {II }} \approx-0.28$ и деформация решетки $\triangle A / A \approx A_{y} / A_{x}-1 \approx-12.6 \%$, если $\Delta a=0$. Вычисленные коэффициенты $C_{\text {I и } C_{\text {II }} \text { указыва- }}$ ют, что наблюдаемое расщепление частот $\Delta \omega$ может быть вызвано анизотропным изменением формы частиц или параметров решетки, при этом величины $\Delta a / a$ и $\Delta A / A$ имеют разные знаки. Расщепление $\Delta \omega_{\|}>0$ может свидетельствовать о сжатии сфероидов или/и о растяжении решетки вдоль оси $x$. С ростом $a / A$ отношение коэффициентов $\left|C_{\mathrm{I}} / C_{\mathrm{II}}\right|$ (величин $|\Delta A / A|$ и $|\Delta a / a|$ ) уменьшается. Следует особо подчеркнуть, что величина $\Delta \omega_{\|}$сильно влияет на вид резонансных спектров $\Delta R / R$ и их масштаб.

\section{8. Заключение}

В работе построена теория анизотропного отражения, обусловленного длинноволновыми коллективными модами дипольных плазмонов, которые возбуждаются в слое наночастиц при нормальном падении света. Показано, что анизотропные плазмонные моды ответственны за формирование резонансной особенности в дифференциальных спектрах отражения, которая исчезает в случае изотропного слоя наночастиц. Сигнал анизотропного отражения, связанный с плазмонами частиц, на два порядка величины больше сигнала, характерного для атомарных поверхностей полупроводников. Представленная теория объясняет, в частности, наблюдавшиеся недавно характерные резонансные спектры анизотропного отражения света в области плазмонных частот нанокластеров индия. Ожидается, что качественные выводы работы применимы к разупорядоченным слоям наночастиц, которые макроскопически однородны и анизотропны в среднем.

Авторы благодарны В.Л. Берковицу за полезные обсуждения и предоставление данных эксперимента.

\section{Список литературы}

[1] Ellipsometry at the Nanoscale / Ed. M. Losurdo, K. Hingerl. Springer. (2013). $730 \mathrm{p}$.

[2] R. Verre, M. Modreanu, O. Ualibek, D. Fox, K. Fleischer, C. Smith, H. Zhang, M. Pemble, J.F. McGilp, I.V. Shvets. Phys. Rev. B 87, 235428 (2013).

[3] В.А. Кособукин. ФТТ 54, 2340 (2012).

[4] N. Esser, A.M. Frisch, A. Roseler, S. Schintke, C. Goletti, D.O. Fimland. Phys. Rev. B 67, 125306 (2003).

[5] В.Л. Берковиц, В.А. Кособукин, В.П. Улин, А.Б. Гордеева, В.Н. Петров. Письма в ЖЭТФ 98, 687 (2013).

[6] V.L. Berkovits, V.A. Kosobukin, V.P. Ulin, A.B. Gordeeva, V.N. Petrov. Surf. Sci. 632, L9 (2015).

[7] R. Verre, K. Fleischer, O. Ualibek, I.V. Shvets. Appl. Phys. Lett. 100, 031102 (2012).

[8] В.Л. Берковиц, А.Б. Гордеева, В.А. Кособукин. ФТТ 43, 985 (2001).

[9] V.L. Berkovits, V.A. Kosobukin, A.B. Gordeeva. J. Appl. Phys. 118, 245305 (2015).

[10] P. Weightman, D.S. Martin, R.J. Cole, T. Farrell. Rep. Prog. Phys. 68, 1251 (2005).

[11] I. Romero, F.J.G. Abajo. Opt. Express 17, 22012 (2009).

[12] L. Persechini, R. Verre, N. McAlinden, J.J. Wang, M. Ranjan, S. Facsko, I.V. Shvets, J.F. McGilp. J. Phys.: Condens. Matter 26, 145302 (2014).

[13] T. Menegotto, F. Horowitz. Appl. Opt. 53, 2853 (2014).

[14] Л.Д. Ландау, Е.М. Лифшиц. Статистическая физика. Наука, М. (1978). Ч. 2. 448 c.

[15] C. Girard, A. Dereux. Rep. Prog. Phys. 59, 657 (1996).

[16] G.C. Schatz. In: Surface Enhanced Raman Scattering / Ed. R.K. Chang, T.E. Furtak. Plenum Press, N.Y. (1982). P. 35.

[17] Л.Д. Ландау, Е.М. Лифшиц. Электродинамика сплошных сред. Наука, М. (1982). 620 с.

[18] B.N.J. Persson, A. Liebsch. Phys. Rev. B 28, 4247 (1983).

[19] H.L. Lemberg, S.A. Rice, D. Guidotti. Phys. Rev. B 10, 4079 (1974).

[20] А.Г. Банщиков, В.Е. Корсуков, В.А. Кособукин. ФТТ 19, 3322 (1977).

[21] F.L. Galeener, G. Lucovsky. Phys. Rev. Lett. 37, 1474 (1976).

[22] R.P. Seisyan, V.A. Kosobukin, S.A. Vaganov, M.A. Markosov, T.S. Shamirzaev, K.S. Zhuravlev, A.K. Bakarov, A.I. Toropov. Phys. Status Solidi C 2, 900 (2005).

[23] R.Y. Koyama, N.V. Smith, W.E. Spicer. Phys. Rev. B 8, 2426 (1973).

[24] D.E. Aspnes, A.A. Studna. Phys. Rev. B 27, 985 (1983). 